\title{
DEVELOPMENT OF ECONOMICAL VEHICLE MODEL FOR PEDESTRIAN-FRIENDLY FRONT-END PROFILE STUDY
}

\author{
Kausalyah, V. ${ }^{*}$; Shasthri, S.**; Abdullah, K. A. ${ }^{* *}$; Idres, M. M. ${ }^{* *}$; Shah, Q. H. ${ }^{* *}$ \& Wong, S. V. ${ }^{* * * *}$ \\ ${ }^{*}$ Faculty of Mechanical Engineering, University Technology MARA, 40450, Selangor, Malaysia \\ ${ }^{* *}$ Department of Mechanical Engineering, Kulliyah of Engineering, International Islamic University \\ Malaysia, 50728 Kuala Lumpur, Malaysia \\ ${ }^{* * *}$ Department of Mechanical Engineering, Faculty of Engineering, University of Selangor, 40450 \\ Shah Alam, Malaysia \\ ${ }^{* * * *}$ Malaysian Institute of Road Safety Research (MIROS), 43000 Kajang, Selangor, Malaysia \\ E-Mail: kausalyah@hotmail.com ('Corresponding author)
}

\begin{abstract}
An economical and deformable, hybrid model is developed for studying the effect of vehicle geometry on pedestrian fall kinematics and associated head injury. A simplified structure consisting of Finite Element surfaces and a Multi-body windshield is built using a series of iterative and non- iterative steps. The primary focus is not so much the stiffness characteristics of the structure, but rather the fall pattern and kinematic data of the pedestrian due solely to the vehicle front-end shape. Comprehensive validation is carried out whereby the fidelity of the model is reviewed for pedestrian crash kinematics and injury criteria as well as piecewise vehicle parts impact tests. The model is shown to hold up acceptably well against benchmarked values especially for the former, whereby very close head injury criteria values are obtained at identical impact locations. The model's notable features are its economical computational processing time and ease of modification.

(Received in October 2013, accepted in March 2014. This paper was with the authors 2 months for 3 revisions.)
\end{abstract}

Key Words: Pedestrian Protection, Deformable Hybrid Vehicle Model, Validation, Optimization Friendly Model

\section{INTRODUCTION}

In motor vehicle-pedestrian crashes, head injuries are frequently the most common injury types, often leading to lifelong disabilities. Statistically, they also record the highest fatality occurrence [1-5]. Much effort has been extended in addressing this problem including law enforcement, increasing awareness, active and passive vehicle safety enhancement, and legislation. In this regard, one effort is the study of the relationship between vehicle front-end shape and the ensuing pedestrian fall pattern and kinematics for improved injury mitigation. About $84 \%$ of all pedestrian fatalities involve frontal impacts, and the vehicle front structure is responsible for most pedestrian injuries [6]. The shape of a vehicle's front-end, traditionally designed according to style, aerodynamics, manufacturability, engine packaging and occupant safety, has been shown to be the most important vehicle design-related factor in determining pedestrian kinematics, which in turn, determines the impact speed, impact angle and location of head impact, ultimately affecting the injury outcome [7, 8]. Parametric studies have been conducted to narrow down the sensitivity of the profile shape dimensions. In the absence of a more appropriate criterion for pedestrian head/brain injuries, commonly a HIC (Head Injury Criterion) with a safety threshold of $\leq 1000$ is used to predict risk of head injury and to assess aggressiveness of the bonnet and windshield in impact simulations [9, 10].

Considering the great cost associated with mechanical optimization of designs and also the limitations of mechanical sub-system impactor tests, computer simulations for studying methods to reduce the loading to a pedestrian in a crash have been on the rise. Earlier optimization works applying Finite Element (FE) modelling were restricted mostly to single 
or dual parameters due to the complexity of the models and the lengthy processing time involved. The advent of Multi-body (MB) modelling technique, though initially developed for studying the gross motion of occupant models during vehicle impact, has evolved to include pedestrian models as well [11]. The advantage of fast modelling and processing speed coupled with model simplicity, despite some limitations in its application, has enabled more exhaustive multi-parametric optimization studies to be carried out [12-14]. Nevertheless, some drawbacks such as the non-deformable nature of the models, curtail the broader application of the exclusive usage of this method. Thus a hybrid modelling method becomes increasingly attractive as it seems to be especially suited for parametric and optimization studies. Hybrid modelling involves the inclusion of rigid bodies (MB) into FE codes allowing seamless integrated Multi-body FE simulations within a common environment. This allows flexibility to use the appropriate modelling methods in unison in view of deriving the maximum advantage of both methods while minimizing the limitations. The technique has seen usage since the 90s [15] and continuous efforts is seen to be underway in crash simulations especially those involving occupants. Leglatin et al. have used in their work the combined multi body and FE technique to model the vehicle with the intention of minimizing the overall computer simulation time and providing realistic predictions in the areas of impact[16]. Ma and Lankarani used hybrid modelling to model crash dynamic responses in occupants in their work. Rigid multi-body dynamics was applied to predict the gross motions and displacements between the rigid bodies and the FE technique was again used to study the structural deformations [17]. Both the studies show that the hybrid modelling technique is able to present an economical and accurate result.

In this study, a deformable vehicle front-end hybrid model built from simple FE profile shapes and a MB plane, is developed and subjected to comprehensive validation. The model is designed to be optimization-friendly i.e. having simple, easily modifiable profile geometry with economical processing time. The intended application of the model is to fulfil the requirements for multi-parametric optimization of vehicle front-end shape, towards mitigation of pedestrian head injuries sustained during impact. The primary focus is not so much the stiffness characteristics of the structure, but rather the fall pattern and kinematic data of the pedestrian due solely to the vehicle front-end shape.

\section{SIMULATION MODELS}

\subsection{Finite Element model}

In studies of motor vehicle crash (MVC) and vehicle-pedestrian impact, it is undeniable that Finite Element (FE) models, when carefully constructed, present unsurpassed accuracy and reliability. However, to preserve these advantages, researches of this nature usually require the building of complex and 'expensive' FE models consisting of numerous parts and connections [18-20]. Despite the availability of FE vehicle and crash-related models such as dummies, impactors and barriers, it nevertheless does not address the overly lengthy pre-processing and processing time [20, 21]. Furthermore, altering model geometry parameters can be quite tedious and difficult to automate. In sensitivity and optimization studies involving multiple parameters, these are all valid concerns to be addressed, and have perhaps helped pave the way for an alternative approach in model building such as the Multi-body method.

\subsection{Multi-body model}

The Multi-body method was developed in the 1980's [22, 23] for the simulation of dynamic behaviour and the gross motion of systems of bodies connected by kinematical joints. This modelling method's usage of rigid planes and ellipsoids with pre-determined contact 
characteristics offers the advantage of unsurpassed processing speed [12, 18, 24] as well as ease of build and validation. It is therefore not surprising that we see the literature being inundated with wide applications of MB models particularly in motor vehicle crash researches $[12,18,25]$.

However, a major drawback associated with this modelling method is the exclusive use of rigid bodies which ignores structural deformation $[19,26]$. Generally, for human models and dummy models, when bio-fidelic considerations are not the focus, structural deformations are less of a factor in determination of fall pattern and most injury criteria. This is largely due to the padding effect afforded by the muscles and tissues of body [12, 19]. Unfortunately, this is not the case for deformable impacting structures such as vehicle models. It would be unrealistic to ignore local deformations of the vehicle body due to impact (see Fig. 1), as it may significantly affect the kinematics of the pedestrian fall and hence the corresponding injury [26]. It can also be argued, that due to the lack of parametric precision in the modelling techniques, multi-body models fall short of being sensitive to small modifications in geometric shape. These postulated drawbacks, although generally acceptable for many types of simulations, nevertheless would prove to be less than ideal in a vehicle front-end shape optimization study.

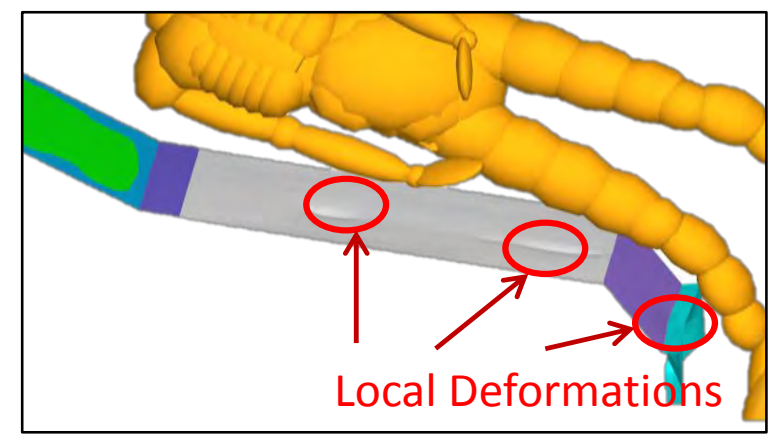

Figure 1: Local deformations upon impact.

\subsection{Hybrid Model}

When conducting pedestrian crash studies with vehicle front-end, particularly with the aim of optimizing the vehicle profile shape, it is necessary to preserve the advantages of both $\mathrm{FE}$ and MB modelling techniques while at the same time minimizing their respective drawbacks. For optimization, the profile geometry needs to be simple enough to allow for quick modifications [20]. It is also necessary for the model to be highly cost effective, both in terms of preprocessing time i.e. model building, as well as processing time $[12,21]$. Additionally, for the intended purpose of the model, it is essential that deformability is retained in the critical parts of the profile in order to capture local deformations.

\section{METHODOLOGY AND MODELLING}

\subsection{Pedestrian ellipsoid model}

MADYMO v7.4.1 by TASS BV is used, due to its support of a hybrid modelling environment, providing tools where both $\mathrm{FE}$ and $\mathrm{MB}$ models can be built and made to interact seamlessly. A commercially available TNO $50^{\text {th }}$ percentile human MB model is used to represent the pedestrian. This model has been extensively validated by TNO and is widely used in vehicle pedestrian research [18, 24, 27-29]. The general injury criteria considered for vehicle shape optimization are the Head Injury Criteria (HIC) and the head centre of gravity 
acceleration $[12,19,26,30]$, although for the purpose of validation, other injury criteria are also referred to $[18,19]$.

\subsection{Vehicle hybrid model}

The development of the simplified vehicle hybrid model, comprise a series of non-iterative and iterative steps. For pedestrian crash kinematics study, the important isolated feature of the bullet vehicle structure is the front-end shape and stiffness. The front-end shape is achieved, whereby similar to a MB modelling technique, a simple planar model representing the frontend profile of a vehicle is built using FE. The advantage here over the MB technique is that the model is able to deform. The stiffness is simulated by means of replicating the material properties and constraints as closely as possible based on the full vehicle system, which are then tuned recursively during validation.

A sample of a vehicle front-end geometry for which there exists a set of validation test data, is adopted from literature [19]. Table I and Fig. 2 show the front-end geometry profile and dimensions. These, as well as the weight, centre of gravity and material properties are maintained as closely as possible, based on the Ford Taurus detailed FE model, developed by EASi Engineering for the National Highway Traffic Safety Administration (NHTSA) [31]. In this study, the HIC values obtained relate to the case where the depth of the hood deformation is smaller than the distance between the hood and the under-hood components. Fig. 3 shows the model which is divided into five parts. A mesh size range of 1 to $5 \mathrm{~mm}$ is used for the vehicle model. Further mesh refinement is found to be unnecessary as it does not significantly alter the HIC results.

Table I. Dimensions of vehicle front-end geometry.

\begin{tabular}{|l|c|}
\hline Part Geometry & Dimension \\
\hline Bumper Height $(B C H)$ & $383 \mathrm{~mm}$ \\
\hline Bumper Lead $(B L)$ & $145 \mathrm{~mm}$ \\
\hline Hood Leading Edge $(H L E)$ height & $763 \mathrm{~mm}$ \\
\hline Hood Length $(H L)$ & $1120 \mathrm{~mm}$ \\
\hline Hood Inclination Angle $(\alpha)$ & $12^{\circ}$ \\
\hline Windshield Inclination Angle $(\beta)$ & $30^{\circ}$ \\
\hline
\end{tabular}

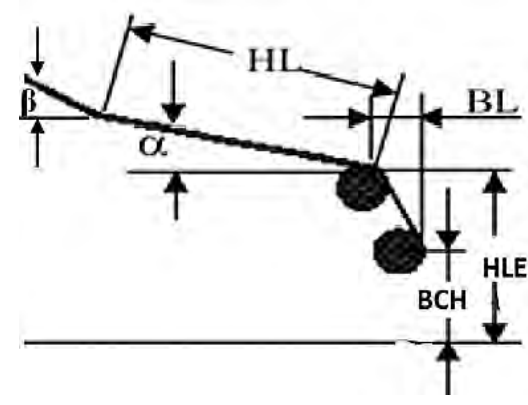

Figure 2: Dimensions of vehicle front-end geometry.

The windshield is made of two layers of tempered glass laminated together with a PVB film layer in between. This is represented in the case model using a MB plane with contact characteristics obtained from Mizuno et al. [32]. Isotropic elasto-plastic material model is used for the cowl, hood, and bumper parts. During pedestrian impact with the vehicle structure, the HLE part is found to have negligible deformation. Thus, following some prevalidation parametric tests, an isotropic linear elastic material model is found to better 
represent the approximate combined higher stiffness of the radiator and front grille associated with the HLE. Table II lists the material properties used for the final model. The use of nonrigid FE surfaces ensures that upon impact, deformations upon the vehicle parts are not inhibited (Fig. 1).

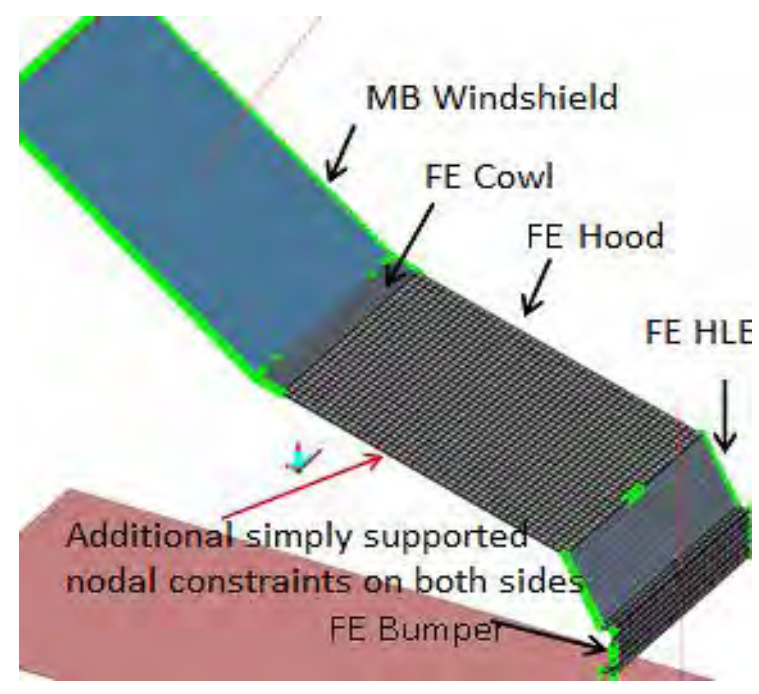

Figure 3: Hybrid model vehicle parts and nodal constraints.

Table II: Material properties for the simplified vehicle profile hybrid model.

\begin{tabular}{|c|c|c|c|c|c|c|}
\hline $\begin{array}{c}\text { Vehicle } \\
\text { parts }\end{array}$ & $\begin{array}{c}\text { Material } \\
\text { model }\end{array}$ & $\begin{array}{c}\text { Density, } \\
\rho \\
\left(\mathrm{kg} / \mathrm{m}^{3}\right)\end{array}$ & $\begin{array}{c}\text { Young's } \\
\text { Mod, } E \\
(\mathrm{GPa})\end{array}$ & $\begin{array}{c}\text { Yield } \\
\text { Stress, } \boldsymbol{\sigma}_{\boldsymbol{y}} \\
(\mathrm{GPa})\end{array}$ & $\begin{array}{c}\text { Poisson's } \\
\text { Ratio, } \mu\end{array}$ & $\begin{array}{c}\text { Quad element } \\
\text { Thickness, } \\
t(\mathrm{~mm})\end{array}$ \\
\hline Cowl & Iso-Plastic & 7800 & 210 & 0.21 & 0.30 & 2.5 \\
\hline Hood & Iso-Plastic & 7800 & 210 & 0.21 & 0.28 & 1.0 \\
\hline HLE & Iso-Linear & 7800 & 210 & - & 0.30 & 3.0 \\
\hline Bumper & Iso-Plastic & 7800 & 200 & 0.1 & 0.35 & 1.5 \\
\hline
\end{tabular}

The bumper, in the interest of simplifying the geometry, is modelled as a single piece comprised of shell elements rather than a multi-part assembly of inner beam, outer plate and connecting parts. The stiffness is lumped together and controlled with material properties and nodal constraints subjected to iterative revision until an acceptable match of the validation criteria is achieved. This is done to merely achieve an approximation of the bumper stiffness magnitude which affects the pedestrian fall and kinematics. A matching stiffness trend however, is not expected, but this is of lesser concern since the study is not tailored towards investigations of lower leg fractures or bumper. All the FE surfaces are attached to a single rigid ellipsoid placed at the centre of gravity which serves as the lumped mass of the vehicle $(1520 \mathrm{~kg})$.

\section{RESULTS AND DISCUSSION}

All the simulations are performed using MADYMO 7.4.1 by TASS on a Lenovo Thinkpad T430 (Intel $2.9 \mathrm{GHz}$ quad core processor, $16 \mathrm{~GB}$ RAM). The simulation run set for a termination time of $250 \mathrm{~ms}$ with time step of $2.0 \mathrm{E}-6$, is found to take only 4 minutes and 18 seconds. Economically comparable with MB models, the Hybrid model is shown to make very significant savings in terms of computational time without sacrificing deformation effects and geometric accuracy. In contrast, an FE model analysis would typically require 
many hours to a day of processing time [20, 21]. Consequently, to assess the optimization feasibility of the proposed simplified vehicle hybrid model, it is subjected to a series of validation tests benchmarked from literature [19, 30]. Validation is accepted based on approximation of curve trend, magnitude, and in pedestrian impact cases, the fall pattern, corresponding injury values especially the HIC and also impact location. Three different validation methods are used as will be discussed.

\subsection{Crash kinematics validation}

This is accepted as the primary validation procedure in determining the fitness of the model. The method here involves a simulation where the hybrid vehicle model is given three different velocities, at $40 \mathrm{~km} / \mathrm{h}$ [19], $32 \mathrm{~km} / \mathrm{h} \mathrm{[33]} \mathrm{and} 25 \mathrm{~km} / \mathrm{h}$ [19], and made to impact a $50^{\text {th }}$ percentile male MB pedestrian as per the methodology outlined in the benchmarked literature. The pedestrian human model is positioned to allow impact to occur on his right side. Gravitational acceleration of $9.81 \mathrm{~ms}^{-2}$ is applied globally to both vehicle and pedestrian. In accordance to IHRA standard, a horizontal braking deceleration is applied to the vehicle at a constant rate of $5 \mathrm{~ms}^{-2}$ [25].

A quantitative (HIC) as well as qualitative (fall pattern based on impact location) assessment is used for the validation and the comparison between the benchmarked values and the case model is presented in Table III. A close agreement is achieved for the corresponding HIC values especially for $25 \mathrm{~km} / \mathrm{h}$ and the case study of $40 \mathrm{~km} / \mathrm{h}$ impact speed. The same impact locations in comparison with the benchmark are also registered. An FE dummy model is used by the benchmark and as such, an exact matching of the validation criteria is not expected. Nevertheless, the comparison is deemed acceptable due to the close similarity of the kinematic impact locations [19, 33]. It should be noted that for the $32 \mathrm{~km} / \mathrm{h}$ impact speed, the value reported by Teng et al. [19] is found to be not in agreement with experimental findings [33] and thus the latter is referred for the benchmark comparison. This may be due to the probability that in the detailed FE vehicle model, there is an occurrence of a secondary contact of the head with rigid under-hood engine parts. However since modern cars are built with sufficient clearance between under-hood components and hood, thus obviating such an occurrence (at standard test velocities at least), the exclusion of this feature in the study is justified.

Table III: Crash kinematics validation results.

\begin{tabular}{|l|c|c|c|}
\hline \multirow{2}{*}{ Criteria } & \multicolumn{3}{|c|}{ Impact velocity $(\mathrm{km} / \mathrm{h})$} \\
\cline { 2 - 4 } & $\mathbf{4 0}$ & $\mathbf{3 2}$ & $\mathbf{2 5}$ \\
\hline HIC Hybrid & 4468 & 1599 & 317 \\
\hline HIC Benchmark & $4795[19]$ & $1469[33]$ & $554[19]$ \\
\hline Impact Location Hybrid & Cowl & Hood & Hood \\
\hline Impact Location Benchmark [19] & Cowl & Hood & Hood \\
\hline
\end{tabular}

Post impact fall pattern images were not reported in the first benchmark. Therefore, for additional kinematic validation, a second benchmark [25] is referred to for comparison. The referred simulation utilizes the same vehicle front-end parameters as used in this study. Furthermore, the pedestrian model used is MB of the same population class. Data is available where post impact kinematic fall patterns are compared for various models at $40 \mathrm{~km} / \mathrm{h}$. Fig. 4 depicts the kinematic impact fall-patterns of this study in comparison with the available data of the second benchmark. It can be seen that for each time frame of $0 \mathrm{~ms}$ to $100 \mathrm{~ms}$, the kinematic fall pattern of the Case model (TNO) is closely comparable with the Post Mortem Human Subjects (PMHS) benchmark. A good match is also seen with the NHTSA dummy 
model. This shows that the case simulation of this study is able to closely simulate the kinematic fall patterns at $40 \mathrm{~km} / \mathrm{h}$ impact speed.

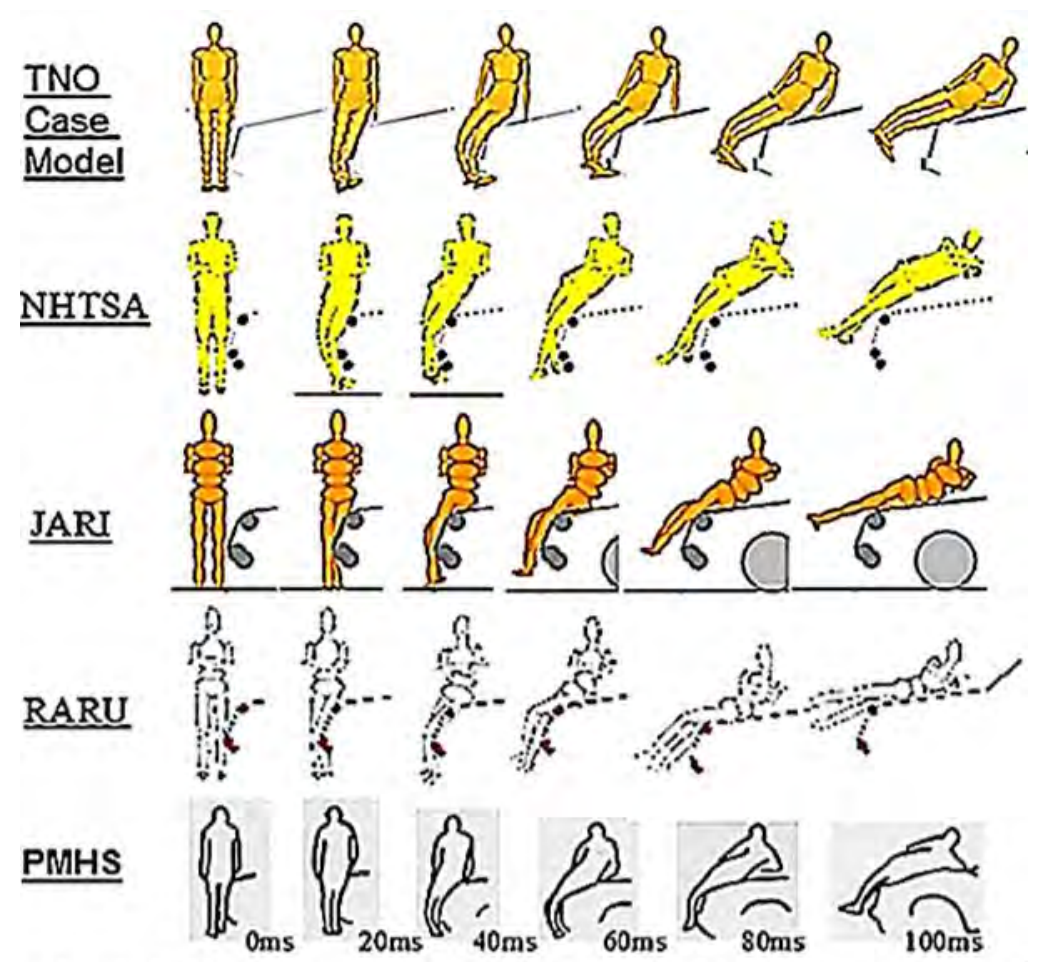

Key: NHTSA = National Highway Traffic Safety Administration JARI = Japan Automobile Research Institue RARU = Road Accident Research Unit of Adelaide University PMHS $=$ Post Mortem Human Subjects

Figure 4: Kinematic post impact fall pattern comparison (figure adopted from [25])

\subsection{Secondary injury criteria validation}

Although the head is made the primary injury criterion for the validation in this study, as additional secondary validation, the hybrid vehicle model is also benchmarked against other pedestrian injury criteria values for pertinent body parts namely the neck, sternum, lumbar, femur and tibia. The same crash kinematics simulation where the vehicle impacts the pedestrian at $40 \mathrm{~km} / \mathrm{h}$ is used to obtain the additional injury output of the pedestrian. This is then compared with similar work from literature [18, 19]. As some injury criteria are generally better represented by FE dummies (benchmarked data), some dissimilarity is expected in the curve trend and peak magnitudes. For conformity, greater emphasis is stressed on the closeness of the peak magnitudes rather than the curve trend. The output signals of the dummy are filtered using the SAE J211/1 sign convention. All accelerations are obtained using the CFC 1000 filter while the force and bending moments are obtained using the CFC 600 filter.

\subsubsection{Neck}

The axial neck force injury criterion is compared for validation as shown in Fig. 5. From the case model plot, it can be seen that all four peaks of the benchmark is reproduced. The first and the third peaks especially are well replicated and are shown to have acceptably close magnitudes with that of the benchmark. Although the second peak registers a lower value, nevertheless, the curve trend is seen to have a close similarity. The fourth peak is also represented adequately. Aggregately, the neck forces registered are found to be acceptable. 


\section{Compressive Neck Forces}

a) Case Model

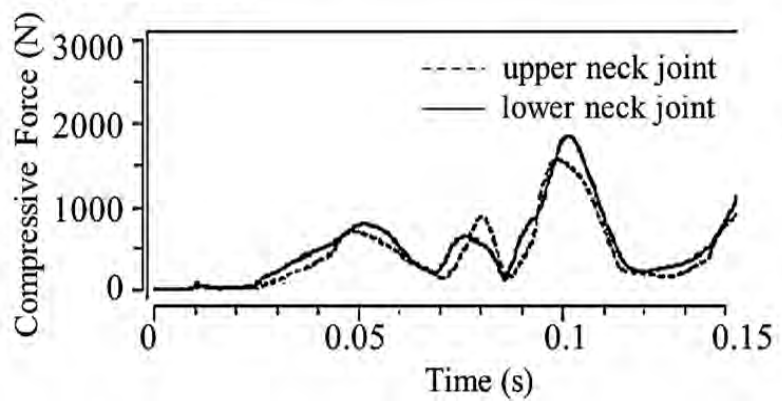

b) Benchmark

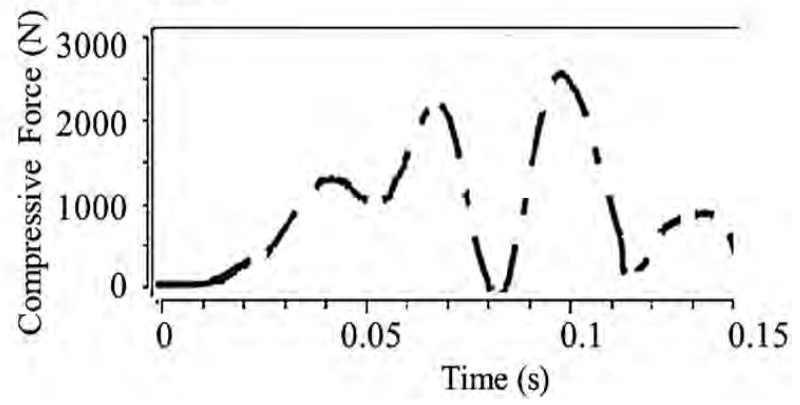

Figure 5: Neck injury criteria - compressive forces plot comparison with benchmark [17].

\subsubsection{Sternum}

Fig. 6 illustrates the resultant chest (sternum) acceleration plot as well as the benchmarked plot. Since chest injury criteria are generally better represented by FE dummies, the dissimilarity of the curve trend is expected and could be attributed to use of the MB dummy in the case model. Alternatively, further tuning of the vehicle hybrid model may possibly give a closer curve match and this would prove to be worthwhile for chest injury severity studies. However, this is assumed to be of lesser concern here in light of the close peak magnitude match obtained. It can be seen that the maximum peak for both the case model as well as the benchmark register an approximate value of $800 \mathrm{~ms}^{-2}$. The results are therefore considered acceptable.

\section{Sternum Acceleration}

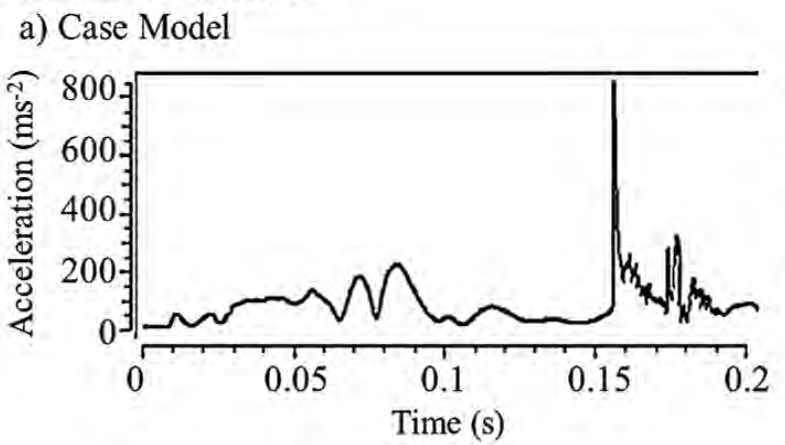

b) Benchmark

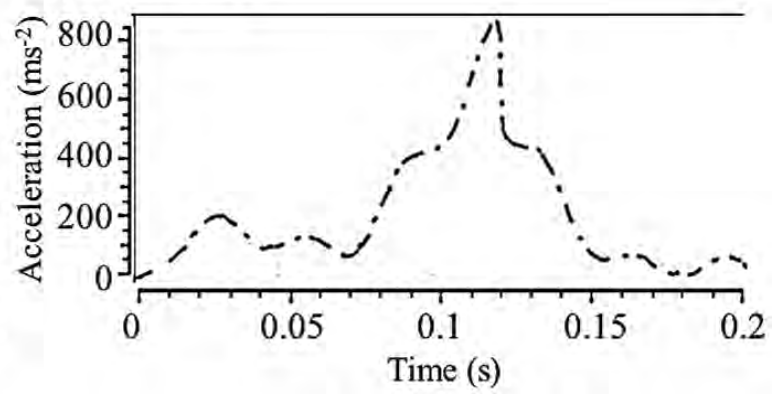

Figure 6: Sternum acceleration - plot comparison with benchmark [17].

\subsubsection{Lumbar}

For lower back injury at the waist joint, the bending moment at the lumbar joint [19] is compared for the model validation as illustrated in Fig. 7. It is evident that a general match is obtained for the curve trend where all three distinctive peaks are reproduced. The maximum value which is achieved by the first peak registers a very close value of approximately 350 $\mathrm{Nm}$ and the third peak is also well replicated with value of less than $200 \mathrm{Nm}$. However, the second peak magnitude is found to be somewhat lower $(160 \mathrm{Nm})$ than the benchmark value $(320 \mathrm{Nm})$. This is attributed largely to secondary contact with under-hood engine parts in the detailed FE vehicle model. Upon impact with the hood, the weight of the dummy which is supported by the lumbar would thus cause the hood to deform largely and affect a secondary combined impact with the under-hood stiff engine parts resulting in the higher second peak. However, in this study, for head injury study, the depth of the hood deformation due to head impact is smaller than the distance between the hood and the under-hood components. 
Therefore the under-hood parts are found not necessary to be included. In light of this, the bending moment obtained is deemed acceptable.

\section{Lumbar Bending Moment}

a) Case Model

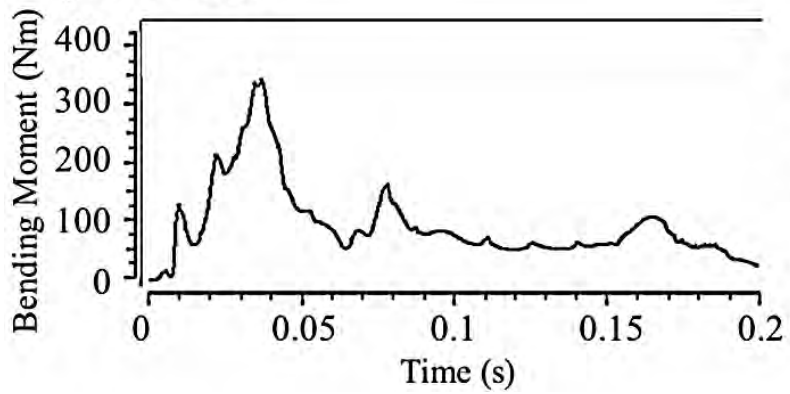

b) Benchmark

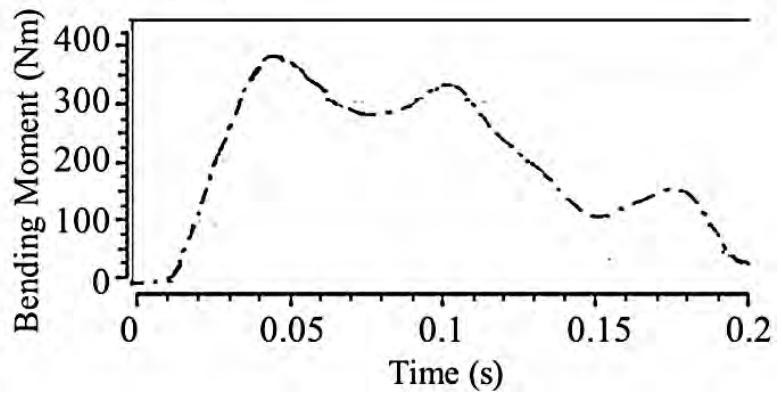

Figure 7: Lumbar bending moment - plot comparison with benchmark [19].

\subsubsection{Lower body (femur and tibia)}

Fig. 8 shows the plot comparison for the femur (thigh bone) bending moment while Fig. 9 depicts the tibia (calf bone) acceleration plot. It is seen that in terms of magnitude a good match is obtained for the Femur Bending Moment with approximate value of $350 \mathrm{Nm}$ for the first peak. The trend is similar although the case model registers lower plateau magnitudes following the initial peak. The tibia acceleration plot also shows good agreement for the first and successive peaks both in terms of magnitude and trend. The difference in time for both Figs. 8 and 9 can be accounted by the damping effect of the bumper assembly which is difficult to reproduce in the simplified model. Although this could not be ignored for lower limb injury study, however for head injury study, these limitations in the case model are acceptable as only stiffness and deformation is required to affect pedestrian kinematics.

\section{Femur Bending Moment}

a) Case Model

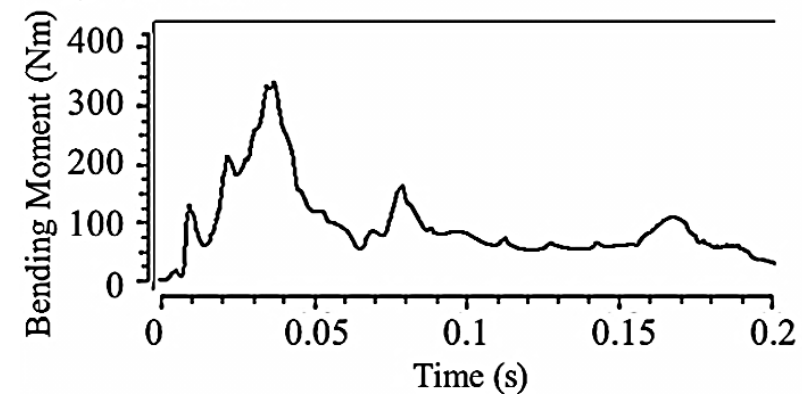

b) Benchmark

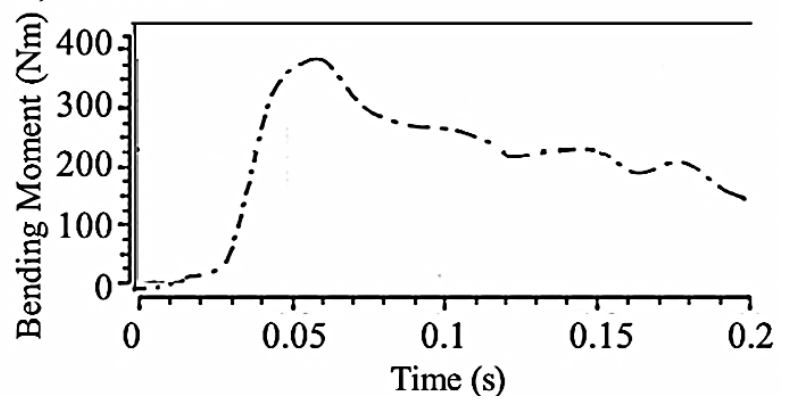

Figure 8: Femur bending moment - plot comparison with benchmark [19].

Tibia Acceleration

a) Case Model

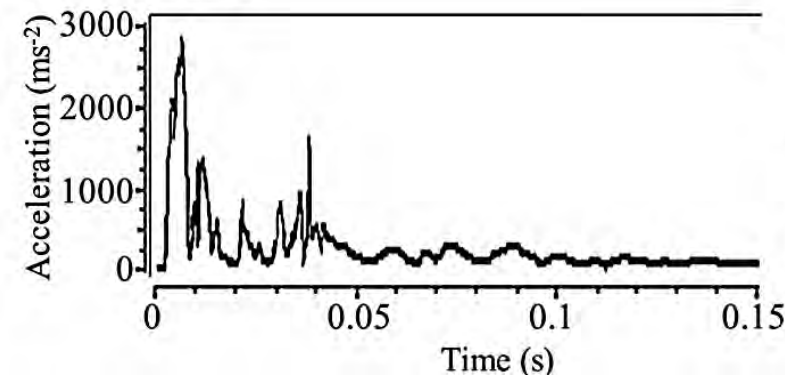

b) Benchmark

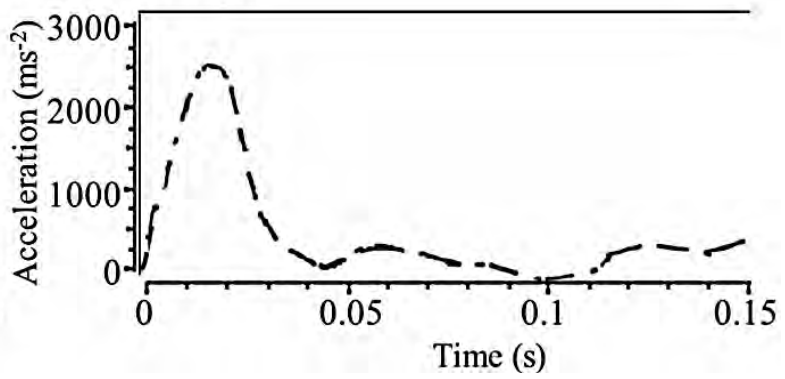

Figure 9: Tibia acceleration - plot comparison with benchmark [19]. 
In summary, it is concluded that with regards to injury criteria validation, the model is found to be acceptable for the required purpose.

\subsection{Piecewise vehicle parts validation}

\subsubsection{Windshield}

In this approach, the vehicle model parts associated with head contact during impact is validated piecewise. By comparing the magnitude and trend, the results serve as a general guideline for additional supplementary model validation.

The MB windshield is impacted perpendicularly with a FE TNO head impactor at $40 \mathrm{~km} / \mathrm{h}$ and compared (Fig. 10) with benchmark data [32, 34, 35] which has been impacted at three different windshield locations, denoted as experiment 1, 2 and 3 (Fig. 10 b). Table IV shows the results obtained and the benchmark comparison. From Fig. 10, it is seen, that both curves register the important initial spike which is caused by the fracturing windshield. After failure, a lower stiffness occurs, which is caused by the stretching of the PVB film layer that holds the broken glass pieces together. The curve trend is found to be similar with that of the benchmark and the peak magnitude difference is a negligible $2.83 \%$.

Table IV: Windshield validation results.

\begin{tabular}{|l|c|c|}
\hline WS results & Head Acc. $\left(\mathrm{m} / \mathrm{s}^{2}\right)$ & HIC15 \\
\hline Hybrid Model & 1233.90 & 260.93 \\
\hline Benchmark [32] & 1200 & - \\
\hline Range $[35,34]$ & $1200-1300$ & $<500$ \\
\hline
\end{tabular}

Windshield - Head Impactor Acceleration Plots

a) Case Model

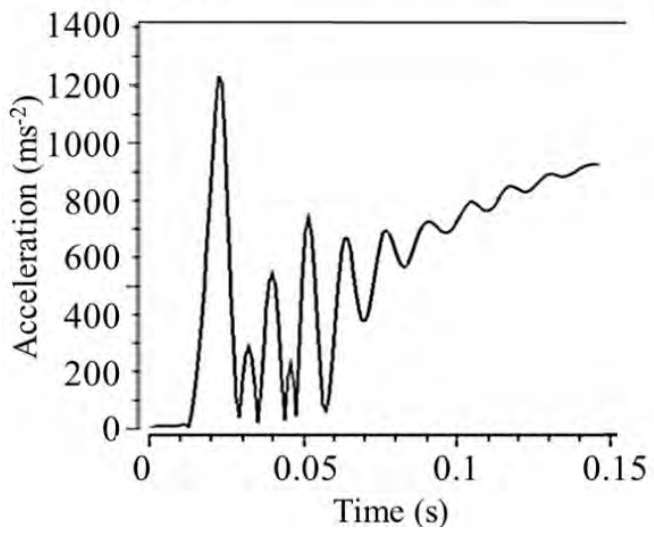

b) Benchmark

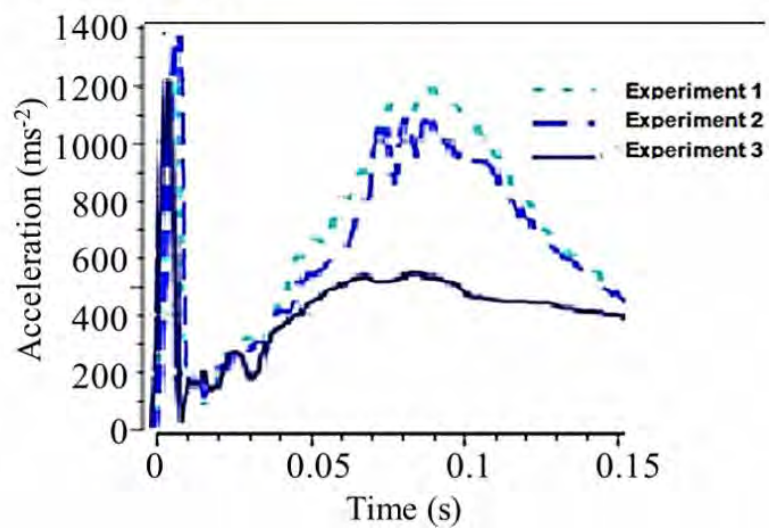

Figure 10: TNO head impactor acceleration plot and benchmark [32] plot for windshield validation.

\subsubsection{Cowl}

The simulation is repeated under identical conditions for cowl validation with the impactor repositioned to impact the cowl perpendicularly [32]. Fig. 11 shows the resulting acceleration plots for the case model and the benchmark, while Table V provides the peak and HIC values for comparison. A good agreement is seen to be achieved for the curve trend in comparison with the benchmarked data, while the peak magnitude difference is acceptably small $(7.14 \%)$. The impact with the cowl registers high head acceleration due to its relative high stiffness and consequently, the resulting HIC value is also large (4581) which agrees well with previous findings [33, 34]. 
Table V: Hood validation results.

\begin{tabular}{|l|c|c|}
\hline Hood results & Head Acc. $\left(\mathrm{m} / \mathrm{s}^{2}\right)$ & HIC15 \\
\hline Hybrid Model & 2990 & 4581 \\
\hline Benchmark [32] & 3300 & - \\
\hline Range $[33,34]$ & - & $3001-5000$ \\
\hline
\end{tabular}

\section{Cowl - Head Impactor Acceleration Plots}

a) Case Model

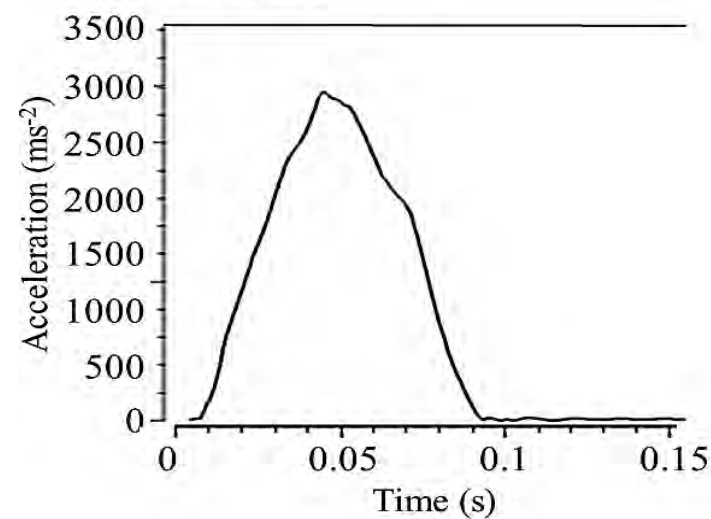

b) Benchmark

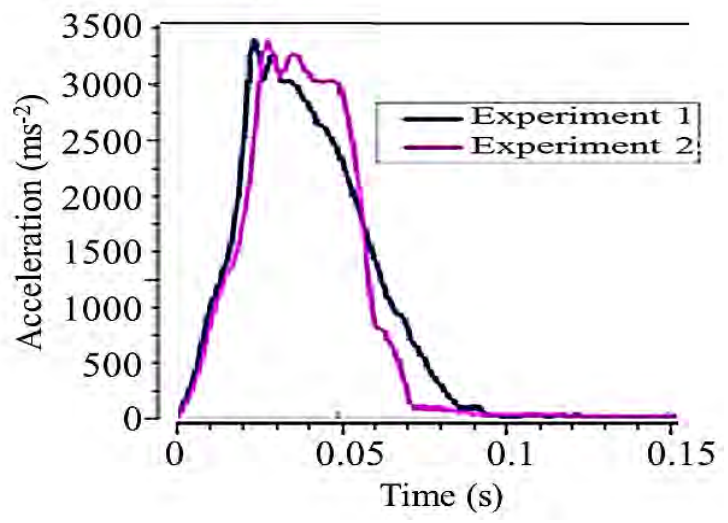

Figure 11: Cowl validation experiment, resulting acceleration plot of the head impactor and benchmark [32].

\subsubsection{Hood}

The hood validation is also performed in a similar manner. The head impactor is repositioned and is made to hit the hood centre region perpendicularly with an initial velocity of $40 \mathrm{~km} / \mathrm{h}$ [32]. Table VI outlines the values and comparisons while the plot trends and peaks can be compared in Fig. 12. The simulation results for the case vehicle give a reasonably good agreement with the benchmark $[32,34,36]$. The peak value is somewhat higher for the case model but is found to be within the acceptable range as is the HIC value. Similarities are also seen in the trend.

Table VI: Hood validation results.

\begin{tabular}{|l|c|c|}
\hline Hood results & Head Acc. $\left(\mathrm{m} / \mathrm{s}^{2}\right)$ & HIC15 \\
\hline Hybrid Model & 1529.7 & 1072.9 \\
\hline Benchmark [32] & 1200 & - \\
\hline Range [36, 34] & $818-2133$ & $500-2000$ \\
\hline
\end{tabular}

Hood - Head Impactor Acceleration Plots

a) Case Model

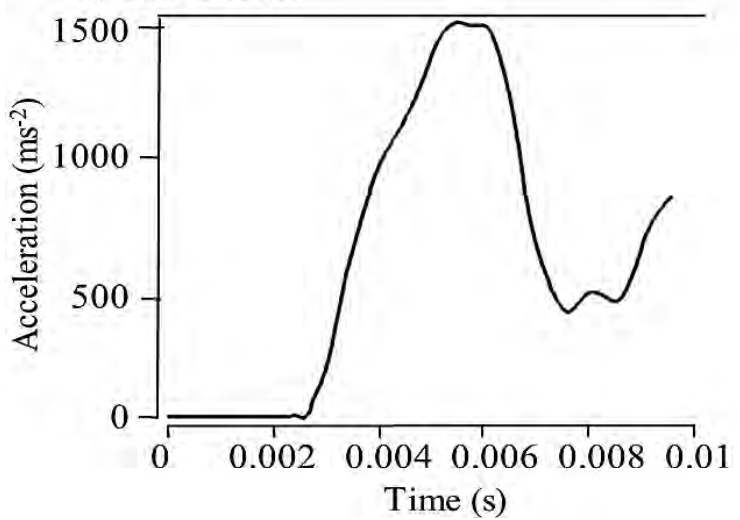

b) Benchmark

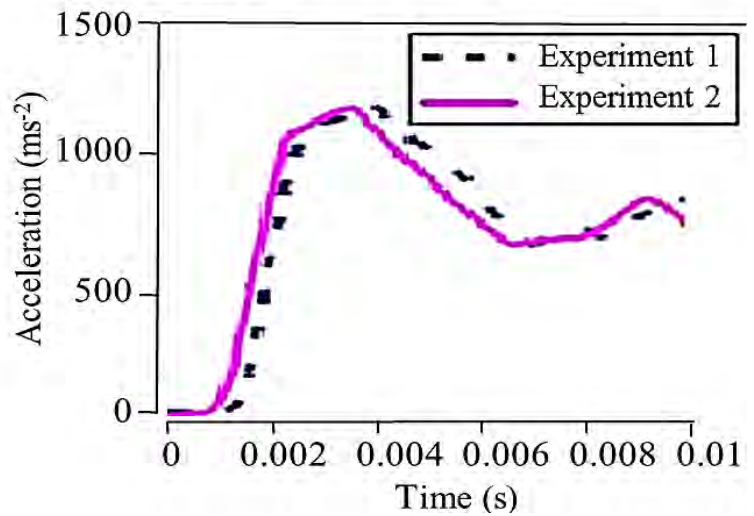

Figure 12: Hood validation acceleration plot of the head impactor and benchmark [32]. 


\section{CONCLUSION}

In summary of the work presented here, the pedestrian crash kinematics, injury criteria and vehicle parts piecewise validations carried out with the vehicle structure is found to show acceptable conformity with the associated benchmarks. The proposed methodology and model offers several advantages in terms of model building time, processing speed, relative accuracy and ease of modifications. In comparison to detailed FE full vehicle or partial vehicle models, fast model building time is achieved due to the use of simple FE planes. Although model tuning during validation trial runs are time consuming, it is not difficult to visualize that the overall model development time frame is found to be much shorter than detailed FE models. A notably short computational processing time is also observed. The simple model constructs allow for quick and easy modifications upon the vehicle geometry thus being highly suited for Design of Experiments approach. In comparison with MB models, although the stated advantages are common to both, however unlike MB models, the deformability of the vehicle structure is preserved, which is necessary for accurate simulation of pedestrian kinematics during impact with vehicle front-end structure. Therefore, it is shown here in this work that the model developed is well suited for multi-parametric study and optimization work towards mitigation efforts of head injury due to pedestrian fall pattern and head impact arising from vehicle collision. In this manner, the methodology presented here, may be employed for quick assessment of any type of vehicle front-end to precisely asses the models pedestrian friendliness.

\section{ACKNOWLEDGEMENT}

The authors would like to thank the Malaysian Ministry of Higher Education (MOHE) and the International Islamic University of Malaysia (IIUM) for their support in awarding the following grants FRGS 12-077-0226 and EDW B11-257-0735 for the purpose of funding this research project.

\section{REFERENCES}

[1] Neal-Sturgess, C. E.; Carter, E; Hardy, R; Cuerden, R; Guerra, L; Yang, J. K. (2007). APROSYS European in-depth pedestrian database, Proceedings of $20^{\text {th }}$ Conference on the Enhanced Safety of Vehicles (ESV), Lyon, Paper Number 07-0267

[2] Peng, Y.; Deck, C.; Yang, J. K.; Cesari, D.; Willinger, R. (2012). A study of kinematics of adult pedestrian and head impact conditions in case of passenger car collisions based on real world accident data, International Research Council on the Biomechanics of Injury (IRCOBI) Conference, Dublin, 766-778

[3] Otte, D. (1999). Severity and mechanism of head impacts in car-to-pedestrian accidents, International Research Council on the Biomechanics of Injury (IRCOBI) Conference, Sitges, 329-341

[4] Maki, T.; Kajzer, J.; Mizuno, K.; Sekine, Y. (2003). Comparative analysis of vehicle-bicyclist and vehicle-pedestrian accidents in Japan, Accident Analysis and Prevention, Vol. 35, No. 6, 927940

[5] Pedestrian Safety Working Group (2001). 2001 Report, International Harmonized Research Activities, Japan Automobile Standards Internationalization Center (JASIC), Japan

[6] Crandall, J. R.; Bhalla, K. S.; Madeley, N. J. (2002). Designing road vehicles for pedestrian protection, British Medical Journal, Vol. 324, No. 7346, 1145-1148

[7] Niederer, P. F; Schlumpf, M. R. (1984). Influence of vehicle front geometry on impacted pedestrian kinematics, SAE Technical Paper 841663, doi:10.4271/841663 
[8] Kuehn, M.; Froeming, R.; Schindler, V. (2003). An advanced testing procedure for the pedestrian-car collision, $18^{\text {th }}$ Int. Technical Conference on the Enhanced Safety of Vehicles, Nagoya, Paper Number 272

[9] EuroNCAP (2009). Pedestrian testing protocol, Version 4.3, from http://www.euroncap.com/files/, accessed on 20-05-2012

[10] European Enhanced Vehicle Safety Committee (1998). EEVC Working Group 17 Report Improved test methods to evaluate pedestrian protection afforded by passenger cars, Brussels

[11] Stammen, J.; Barsan-Anelli, A. (2001). Adaptation of a human body mathematical model to simulation of pedestrian/vehicle interactions, $4^{\text {th }}$ MADYMO User's Meeting of the America's, Detroit

[12] Carter, E; Ebdon, S.; Neal-Sturgess, C. (2005). Optimization of passenger car design for the mitigation of pedestrian head injury using a genetic algorithm, Proceedings of the 2005 Conference on Genetic and evolutionary computation, New York, 2113-2120

[13] Linder, A.; Clark, A.; Douglas, C.; Fildes, B.; Yang, J.; Sparke, L. (2004). Mathematical modelling of pedestrian crashes: Review of pedestrian models and parameter study of the influence of the sedan vehicle contour, Proceedings of the Australasian road safety research, policing and education conference, Vol. 8, No. 2

[14] Zhao, Y.; Rosala, G. F.; Campean, I. F.; Day, A. J. (2010). A response surface approach to frontcar optimisation for minimising pedestrian head injury levels, International Journal of Crashworthiness, Vol. 15, No. 2, 143-150, doi:10.1080/13588260903094392

[15] Bruijs, W. E. M. (1990). Subcycling in transient finite element analysis, PhD Thesis, Department of Mechanical Engineering, Eindhoven University of Technology, Eindhoven

[16] Leglatin, N.; Blundell, M. V.; Blount, G. N. (2006). The simulation of pedestrian impact with a combined multibody finite elements system model, Journal of Engineering Design, Vol. 17, No. 5, 463-477, doi:10.1080/09544820600664622

[17] Ma, D.; Lankarani, H. M. (1997). A multibody/finite element analysis approach for modeling crash dynamic responses, Journal of Mechanical Design, Vol. 119, No. 3, 382-387, doi: $10.1115 / 1.2826359$

[18] Rooij, L.; Bhalla, K.; Meissner, M.; Ivarsson, J.; Crandall, J.; Longhitano, D.; Takahashi, Y.; Dokko, Y.; Kikuchi, Y. (2003). Pedestrian crash reconstruction using multi-body modelling with geometrically detailed, validated vehicle models and advanced pedestrian injury criteria, Proceedings of $19^{\text {th }}$ Int. Technical Conference on the Enhanced Safety of Vehicles, Nagoya, Paper No. 468

[19] Teng, T. L.; Le, T. K.; Ngo, V. L. (2010). Injury analysis of pedestrians in collisions using the pedestrian deformable model, International Journal of Automotive Technology, Vol. 11, No. 2, $187-195$

[20] Kurtaran, H.; Eskandarian, A.; Marzougui, D.; Bedewi, N. E. (2002). Crashworthiness design optimization using successive response surface approximations, Computational Mechanics, Vol. 29, No. 4-5, 409-421, doi:10.1007/s00466-002-0351-x

[21] Sun, G.; Li, G.; Zhou, S.; Li, H.; Hou, S.; Li, Q. (2011). Crashworthiness design of vehicle by using multi-objective robust optimization, Structural and Multidisciplinary Optimization, Vol. 44, No. 1, 99-110, doi:10.1007/s00158-010-0601-z

[22] Huston, R. L.; Passerello, C. E.; Harlow, M. W. (1978). Dynamics of multi-rigid-body systems, Journal of Applied Mechanics, Vol. 45, No. 4, 889-894

[23] Kane, T. R.; Levinson, D. A. (1985). Dynamics: Theory and applications, McGraw-Hill, New York

[24] de Lange, R.; van Rooij, L.; Happee, R.; Liu, X. J. (2006). Validation of human pedestrian models using laboratory data as well as accident reconstruction, Expert Symposium on Accident Research, Hannover, 186-193

[25] Mizuno, Y. (2003). Summary of IHRA pedestrian safety working group activities 2003 Proposed test methods to evaluate pedestrian protection afforded by passenger cars, $18^{\text {th }}$ Int. Technical Conference on the Enhanced Safety of Vehicles, Nagoya, Paper Number 580

[26] Liu, X. J.; Yang, J. (2003), Effects of vehicle impact velocity and front-end structure on dynamic responses of child pedestrians, Traffic Injury Prevention, Vol. 4, No. 4, 337-344, doi:10.1080/ $\underline{714040491}$ 
[27] van Hoof, J.; de Lange, R.; Wismans, J. (2003), Improving pedestrian safety using numerical human models, Stapp Car Crash Journal, Vol. 47, 401-436

[28] TNO Automotive (2006). MADYMO Human Models Manual, TNO Automotive, Delft

[29] Yang, J. K.; Lovsund, P.; Cavalerro, C.; Bonnoit, J. (2000). A human body 3D mathematical model for simulation of car-pedestrian impacts, Journal of Crash Prevention and Injury Control, Vol. 2, No. 2, 131-149, doi:10.1080/10286580008902559

[30] Mizuno, K.; Yonezawa, H.; Kajzer, J. (2001). Pedestrian headform impact tests for various vehicle locations, $17^{\text {th }}$ Int. Technical Conference on the Enhanced Safety of Vehicles, Amsterdam, Paper Number 278

[31] $\mathrm{Wu}, \mathrm{C} . \mathrm{C}$. (2003). The analysis of occupant injury in frontal impact of traffic accident, M.S. Thesis, Da-Yeh University, Taiwan

[32] Mizuno, K.; Kajzer, J. (2000). Head injuries in vehicle-pedestrian impact, SAE Paper No. 200001-0157, Proceedings of the Society of Automotive Engineers 2000 World Congress, Detroit

[33] Anata, K.; Konosu, A.; Issiki, T. (2011). Injury risk assessment at the timing of pedestrian impact with a road surface in a car-pedestrian accident, $22^{\text {nd }}$ Int. Technical Conference on the Enhanced Safety of Vehicles, Washington, DC, Paper No. 11-0119

[34] Mizuno, K.; Yonezawa, H.; Kajzer, J. (2001). Pedestrian headform impact tests for various vehicle locations, SAE Paper No. 2001-06-0185, Society of Automotive Engineers, Warrendale

[35] Yong, P.; Deck, C.; Yang, J.; Willinger, R. (2012). Modelling and validation of windscreen laminated glass behaviour during fracture, Third International Conference on Digital Manufacturing and Automation, Guilin, China, 541-544

[36] Krishnamoorthy, R.; Takla, M.; Subic, A.; Scott, D. (2013). Design optimization of passenger car hood panels for improved pedestrian protection, Advanced Materials Research, Vol. 633, 62-76, doi:10.4028/www.scientific.net/AMR.633.62 